Journal of Universal Mathematics

VOL.2 No.1 PP.75-81 (2019)

ISSN-2618-5660

\title{
INEXTENSIBLE FLOWS OF CURVES ACCORDING TO SABBAN FRAME IN GALILEAN SPACE $G_{3}$
}

\author{
HÜLYA GÜN BOZOK
}

\begin{abstract}
In this paper inextensible flows of curves in 3-dimensional Galilean space is researched. Firstly Sabban frame is defined in 3-dimensional Galilean space, then necessary and sufficient conditions for inextensible flows of curves with Sabban frame in 3-dimensional Galilean space are given. Also inextensible curve flow are expressed as a partial differential equation involving geodesic curvature according to this frame.
\end{abstract}

\section{INTRODUCTION}

The flow of a curve is said to be inextensible if, in the former case, its arclength is preserved, and in the latter case, if its intrinsic curvature is preserved. Physically, the inextensible curve flows give rise to motions in which no strain energy is induced. Such that a piece of paper carried by the wind, can be described by inextensible curve and surface flows. These motions used physical applications [10, 12] and also arise in computer vision $[18,13]$, computer animation [19] and even structural mechanics [7].

The distinction between heat flows and inextensible flows of planar curves are researched in detail, and some examples of the latter are given in [6]. Also, a general formulation for inextensible flows of curves and developable surfaces in $\mathbb{R}^{3}$ are exposed by [5]. Latifi et al. [8] studied inextensible flows of curves in Minkowski 3-space. Moreover Öğrenmiş et al. [2] studied inextensible curves in the Galilean space $G_{3}$, Ergüt et al.[17] researched characterization of inextensible flows of spacelike curves with Sabban Frame in $S_{1}^{2}$ and Öztekin et al. [11] investigated inextensible flows of curves according to Sabban frame in pseudo-Galilean Space.

A Galilean space may be considered as the limit case of a pseudo-Euclidean space in which the isotropic cone degenerates to a plane. On the study of a Galilean space, B. Divjak and M. Sipus [4] investigated the properties of helical surfaces, ruled screw surfaces and rotation surface in 3-dimensional Galilean space $G_{3}$. Ögrrenmiş studied helices in 3-dimensional Galilean space $G_{3}[1]$. Also, Öğrenmiş studied the Frenet formula and Mannheim curve of AW( $k$-type in pseudo-Galilean space [3].

In this paper we give Sabban frame in Galilean space $G_{3}$ and research inextensible flows of curves according to Sabban frame in Galilean space $G_{3}$. Then we obtain

Date: Review January 2, 2019, accepted January 14, 2019.

2000 Mathematics Subject Classification. 53A35.

Key words and phrases. Inextensible flows, Sabban Frame, Galilean Space. 
partial differential equations in terms of inextensible flows of curves with this frame in $G_{3}$.

\section{Preliminaries}

The Galilean space $G_{3}$ is a Cayley-Klein space equipped with the projective metric of signature $(0,0,+,+)$ as in $[9,21]$. The absolute figure of Galilean geometry consist of an ordered triple $\{w, f, I\}$, where $w$ is the ideal (absolute) plane, $f$ is the line (absolute line) in $w$ and $I$ is the fixed elliptic involution of points of $f$.

Galilean scalar product can be written as

$$
\left\langle v_{1}, v_{2}\right\rangle= \begin{cases}x_{1} x_{2} & \text {, if } x_{1} \neq 0 \vee x_{2} \neq 0 \\ y_{1} y_{2}+z_{1} z_{2} & \text {, if } x_{1}=0 \wedge x_{2}=0\end{cases}
$$

where $v_{1}=\left(x_{1}, y_{1}, z_{1}\right)$ and $v_{2}=\left(x_{2}, y_{2}, z_{2}\right)$. It leaves invariant the Galilean norm of the vector $v=(x, y, z)$ defined by

$$
\|v\|= \begin{cases}x & , \text { if } x \neq 0 \\ \sqrt{y^{2}+z^{2}} & , \text { if } x=0\end{cases}
$$

[20]. If a curve $C$ of the class $C^{r}(r \geq 3)$ is given by the parametrization

$$
r=r(x, y(x), z(x))
$$

then $x$ is a Galilean invariant the arc length on $C$. The curvature is

$$
\kappa(x)=\sqrt{y^{\prime \prime 2}+z^{\prime \prime 2}}
$$

and torsion

$$
\tau(x)=\frac{1}{\kappa^{2}(x)} \operatorname{det}\left(r^{\prime}(x), r^{\prime \prime}(x), r^{\prime \prime \prime}(x)\right)
$$

The orthonormal trihedron is defined

$$
\begin{aligned}
t(x) & =\left(1, y^{\prime}(x), z^{\prime}(x)\right) \\
n(x) & =\frac{1}{\kappa(x)}\left(0, y^{\prime \prime}(x), z^{\prime \prime}(x)\right) \\
b(x) & =\frac{1}{\kappa(x)}\left(0,-z^{\prime \prime}(x), y^{\prime \prime}(x)\right)
\end{aligned}
$$

The vectors $t, n, b$ are called the vectors of tangent, principal normal and binormal line of, respectively. For their derivatives the following Frenet formulas hold

$$
\begin{aligned}
t^{\prime}(x) & =\kappa(x) n(x) \\
n^{\prime}(x) & =\tau(x) b(x) \\
b^{\prime}(x) & =-\tau(x) n(x)
\end{aligned}
$$

[15]. For detailed information abot galilean geometry we refer to [20, 14].

Now we give a new frame different from Frenet frame. Let $\gamma: I \rightarrow S^{2}$ be a unit speed spherical curve. In this section we denote $\sigma$ as the arc-length parameter of $\gamma$. Let us denote $t(\sigma)=\dot{\gamma}(\sigma)$, and we call $t(\sigma)$ a unit tangent vector of $\gamma$ at $\sigma$, where $\dot{\gamma}=\frac{d \gamma}{d \sigma}$. We now set a vector $s(\sigma)=\gamma(\sigma) \times t(\sigma)$. By definition we have an orthonormal frame $\{\gamma(\sigma), t(\sigma), s(\sigma)\}$ along $\gamma$. This frame is called Sabban frame of $\gamma[16]$. 
Theorem 2.1. Let $\gamma: I \rightarrow S_{G}^{2}$ be a unit speed spherical curve in Galilean 3-space. We denote $\sigma$ as the arc-length parameter of $\gamma$. Then we have the following spherical Frenet-Serret formulae of $\gamma$ :

$$
\begin{aligned}
& \dot{\gamma}(\sigma)=t(\sigma) \\
& \dot{t}(\sigma)=-\gamma(\sigma)+\kappa_{g}(\sigma) s(\sigma) \\
& \dot{s}(\sigma)=-\kappa_{g}(\sigma) t(\sigma)
\end{aligned}
$$

where $\kappa_{g}(\sigma)$ is the geodesic curvature of the curve $\gamma$ in $S_{G}^{2}$ which is given by $\kappa_{g}(\sigma)=$ $\operatorname{det}(\gamma(\sigma), t(\sigma), \dot{t}(\sigma))$ or

$$
\begin{aligned}
\kappa_{g}(\sigma) & =\operatorname{det}(\gamma(\sigma), t(\sigma), \dot{t}(\sigma)) \\
& =\langle\gamma(\sigma) \times t(\sigma), \dot{t}(\sigma)\rangle \\
& =\langle s(\sigma), \dot{t}(\sigma)\rangle
\end{aligned}
$$

where $\langle\dot{\gamma}(\sigma), t(\sigma)\rangle=1$.

\section{Inextensible Flows of Curves According to Sabban Frame In Galilean Space $G_{3}$}

Throughout this paper, we assume that $\gamma(u, t)$ is a one parameter family of smooth curves in Galilean space $G_{3}$. The arclength of $\gamma$ is given by

$$
\sigma(u)=\int_{0}^{u}\left|\frac{\partial \gamma}{\partial u}\right| d u
$$

where

$$
\left|\frac{\partial \gamma}{\partial u}\right|=\left|\left\langle\frac{\partial \gamma}{\partial u}, \frac{\partial \gamma}{\partial u}\right\rangle\right|^{\frac{1}{2}}
$$

The operator $\frac{\partial}{\partial \sigma}$ is given in terms of $u$ by

$$
\frac{\partial}{\partial \sigma}=\frac{1}{v} \frac{\partial}{\partial u}
$$

where $v=\left|\frac{\partial \gamma}{\partial u}\right|$ and the arclength parameter is $d \gamma=v d u$. Any flow of $\gamma$ can be represented as

$$
\frac{\partial \gamma}{\partial t}=f \gamma+g t+h s
$$

Letting the arclength variation be

$$
\sigma(u, t)=\int_{0}^{u} v d u
$$

In the Galilean space $G_{3}$ the requirement that the curve not be subject to any elongation or compression can be expressed by the condition

$$
\frac{\partial}{\partial t} \sigma(u, t)=\int_{0}^{u} \frac{\partial v}{\partial t} d u=0
$$

for all $u \in[0, l]$. 
Definition 3.1. A curve evolution $\gamma(u, t)$ and its flow $\frac{\partial \gamma}{\partial t}$ in Galilean space $G_{3}$ are said to be inextensible if

$$
\frac{\partial}{\partial t}\left|\frac{\partial \gamma}{\partial u}\right|=0
$$

Lemma 3.2. Let $\frac{\partial \gamma}{\partial t}=f \alpha+g t+h$ s be a smooth flow of the curve $\gamma$ in Galilean space $G_{3}$. The flow is inextensible if and only if

$$
\frac{\partial v}{\partial t}=\frac{\partial g}{\partial u}+f v-h v \kappa_{g} .
$$

Proof. Suppose that $\frac{\partial \gamma}{\partial t}$ be a smooth flow of the curve $\gamma$ in Galilean space $G_{3}$. From (3.2), we obtain

$$
v \frac{\partial v}{\partial t}=\left\langle\frac{\partial \gamma}{\partial u}, \frac{\partial}{\partial u}(f \gamma+g t+h s)\right\rangle .
$$

By the formula of the Sabban, we have

$$
\frac{\partial v}{\partial t}=\left\langle t,\left(\frac{\partial f}{\partial u}-g v\right) \gamma+\left(\frac{\partial g}{\partial u}+f v-h v \kappa_{g}\right) t+\left(\frac{\partial h}{\partial u}+g v \kappa_{g}\right) s\right\rangle .
$$

Making necessary calculations from above equation, we have (3.4), which proves the lemma.

Theorem 3.3. Let $\frac{\partial \gamma}{\partial t}=f \gamma+g t+h$ s be a smooth flow of the curve $\gamma$ in Galilean space $G_{3}$ The flow is inextensible if and only if

$$
\frac{\partial g}{\partial u}=h v \kappa_{g}-f v
$$

Proof. From (3.3), we have

$$
\frac{\partial}{\partial t} \sigma(u, t)=\int_{0}^{u} \frac{\partial v}{\partial t} d u=\int_{0}^{u}\left(f v+\frac{\partial g}{\partial u}-h v \kappa_{g}\right)=0 .
$$

Substituting (3.4) in (3.6) complete the proof of the theorem.

We now restrict ourselves to arc length parametrized curves. That is, $v=1$ and the local coordinate $u$ corresponds to the curve arc length $\sigma$. We require the following lemma.

Lemma 3.4. Let $\frac{\partial \gamma}{\partial t}=f \gamma+g t+h$ s be a smooth flow of the curve $\gamma$ in Galilean space $G_{3}$. Then,

$$
\begin{aligned}
\frac{\partial \gamma}{\partial t} & =-\left(\frac{\partial f}{\partial \sigma}-g\right) t+\varphi s \\
\frac{\partial t}{\partial t} & =\left(\frac{\partial f}{\partial \sigma}-g\right) \gamma+\left(\frac{\partial h}{\partial \sigma}+g \kappa_{g}\right) s \\
\frac{\partial s}{\partial t} & =-\left(\frac{\partial h}{\partial \sigma}+g \kappa_{g}\right) t-\varphi \gamma
\end{aligned}
$$

where $\varphi=\left\langle\frac{\partial \gamma}{\partial t}, s\right\rangle$. 
Proof. Using definition of $\gamma$, we get

$$
\frac{\partial t}{\partial t}=\frac{\partial}{\partial t} \frac{\partial \gamma}{\partial \sigma}=\frac{\partial}{\partial \sigma}(f \gamma+g t+h s) .
$$

Using the Sabban frame, we obtain

$$
\frac{\partial t}{\partial t}=\left(\frac{\partial f}{\partial \sigma}-g\right) \gamma+\left(\frac{\partial g}{\partial \sigma}+f-h \kappa_{g}\right) t+\left(\frac{\partial h}{\partial \sigma}+g \kappa_{g}\right) s
$$

On the other hand substituting (3.5) in (3.8), we get

$$
\frac{\partial t}{\partial t}=\left(\frac{\partial f}{\partial \sigma}-g\right) \gamma+\left(\frac{\partial h}{\partial \sigma}+g \kappa_{g}\right) s
$$

Also we have,

$$
\begin{aligned}
0 & =\frac{\partial}{\partial t}\langle t, \gamma\rangle=\frac{\partial f}{\partial \sigma}-g+\left\langle t, \frac{\partial \gamma}{\partial t}\right\rangle, \\
0 & =\frac{\partial}{\partial t}\langle t, s\rangle=\frac{\partial h}{\partial \sigma}+g \kappa_{g}+\left\langle t, \frac{\partial s}{\partial t}\right\rangle, \\
0 & =\frac{\partial}{\partial t}\langle\gamma, s\rangle=\varphi+\left\langle\gamma, \frac{\partial s}{\partial t}\right\rangle .
\end{aligned}
$$

Then, a straight forward computation using above system gives

$$
\begin{aligned}
& \frac{\partial \gamma}{\partial t}=-\left(\frac{\partial f}{\partial \sigma}-g\right) t+\varphi s \\
& \frac{\partial s}{\partial t}=-\left(\frac{\partial h}{\partial \sigma}+g \kappa_{g}\right) t-\varphi \gamma
\end{aligned}
$$

where $\varphi=\left\langle\frac{\partial \gamma}{\partial t}, s\right\rangle$. Thus, we obtain the theorem.

The following theorem states the conditions on the curvature and torsion for the flow to be inextensible.

Theorem 3.5. Let $\frac{\partial \gamma}{\partial t}$ is inextensible. Then, the following system of partial differential equations holds:

$$
\begin{aligned}
\frac{\partial \kappa_{g}}{\partial \sigma}-\varphi & =\frac{\partial}{\partial \sigma}\left(g \kappa_{g}\right)+\frac{\partial^{2} h}{\partial \sigma^{2}} \\
\kappa_{g} \varphi & =-\frac{\partial^{2} f}{\partial \sigma^{2}}+\frac{\partial g}{\partial \sigma} .
\end{aligned}
$$

Proof. Using (3.7), we have

$$
\begin{aligned}
\frac{\partial}{\partial \sigma} \frac{\partial t}{\partial t}= & \frac{\partial}{\partial \sigma}\left[\left(\frac{\partial f}{\partial \sigma}-g\right) \gamma+\left(\frac{\partial h}{\partial \sigma}+g \kappa_{g}\right) s\right] \\
= & \left(\frac{\partial^{2} f}{\partial \sigma^{2}}-\frac{\partial g}{\partial \sigma}\right) \gamma+\left[\left(\frac{\partial f}{\partial \sigma}-g\right)-\kappa_{g}\left(\frac{\partial h}{\partial \sigma}+g \kappa_{g}\right)\right] t \\
& +\left(\frac{\partial^{2} h}{\partial \sigma^{2}}+\frac{\partial}{\partial \sigma}\left(g \kappa_{g}\right)\right) s
\end{aligned}
$$


On the other hand, considering Sabban frame we obtain

$$
\begin{aligned}
\frac{\partial}{\partial \sigma} \frac{\partial t}{\partial t}= & \frac{\partial}{\partial t}\left(-\gamma+\kappa_{g} s\right) \\
= & \left(\frac{\partial \kappa_{g}}{\partial \sigma}-\varphi\right) s+\left[\left(\frac{\partial f}{\partial \sigma}-g\right)+\kappa_{g}\left(-\frac{\partial h}{\partial \sigma}-g \kappa_{g}\right)\right] t \\
& -\kappa_{g} \varphi \alpha
\end{aligned}
$$

Hence we see that

and

$$
\frac{\partial \kappa_{g}}{\partial \sigma}-\varphi=\frac{\partial}{\partial \sigma}\left(g \kappa_{g}\right)+\frac{\partial^{2} h}{\partial \sigma^{2}}
$$

Thus we obtain the theorem.

$$
\kappa_{g} \varphi=-\frac{\partial^{2} f}{\partial \sigma^{2}}+\frac{\partial g}{\partial \sigma}
$$

Theorem 3.6. Let $\frac{\partial \gamma}{\partial t}=f \gamma+g t+h$ s be a smooth flow of the curve $\alpha$ in Galilean space $G_{3}$. Then,

$$
\kappa_{g}\left(\frac{\partial f}{\partial \sigma}-g\right)=-\left(\frac{\partial h}{\partial \sigma}+h \kappa_{g}\right)+\frac{\partial \varphi}{\partial \sigma}
$$

Proof. Similarly, we have

$$
\begin{aligned}
\frac{\partial}{\partial \sigma} \frac{\partial s}{\partial t}= & \frac{\partial}{\partial \sigma}\left[\left(-\frac{\partial h}{\partial \sigma}-g \kappa_{g}\right) t-\varphi \alpha\right] \\
= & \left(-\frac{\partial^{2} h}{\partial \sigma^{2}}-\frac{\partial}{\partial \sigma}\left(g \kappa_{g}\right)-\varphi\right) t-\kappa_{g}\left(\frac{\partial h}{\partial \sigma}+g \kappa_{g}\right) s \\
& +\left(\frac{\partial h}{\partial \sigma}+g \kappa_{g}-\frac{\partial \varphi}{\partial \sigma}\right) \alpha
\end{aligned}
$$

Then we get

$$
\begin{aligned}
\frac{\partial}{\partial t} \frac{\partial s}{\partial \sigma} & =\frac{\partial}{\partial t}\left(-\kappa_{g} t\right) \\
& =-\frac{\partial \kappa_{g}}{\partial t} t-\kappa_{g}\left[\left(\frac{\partial f}{\partial \sigma}-g\right) \alpha+\left(\frac{\partial h}{\partial \sigma}+g \kappa_{g}\right) s\right]
\end{aligned}
$$

So using above theorem we give the following corollary without proof:

\section{Corollary 3.7.}

$$
\varphi=-\frac{\partial^{2} h}{\partial \sigma^{2}}-\frac{\partial}{\partial \sigma}\left(g \kappa_{g}\right)+\frac{\partial \kappa_{g}}{\partial t}
$$

\section{REFERENCES}

[1] A. O. Öğrenmiş, M. Bektaş, M. Ergüt, On the helices in the Galilean space $G_{3}$, Iranian Journal of science \& Technology, Transaction A, 31, 177-181, (2007).

[2] A. O. Öğrenmiş, M. Yeneroğlu, Inextensible curves in the Galilean space, International Journal of the Physical Sciences, 5(9),1424-1427,(2010).

[3] A. O. Öğrenmiş, On curvatures of a frenet curve in the pseudo-Galilean space $G_{1}^{3}$ Inter. J. Phys.Sci. 5, 2363-2365, (2010).

[4] B. Divjak and M. Sipus, Some special surfaces in the pseudo-Galilean space,Acta Math. Hungar. 118, 209-229,(2008). 
[5] D. Y. Kwon, FC. Park, DP Chi, Inextensible flows of curves and developable surfaces, Applied Mathematics Letters 18, 1156-1162, (2005).

[6] D.Y. Kwon, F.C. Park, , Evolution of inelastic plane curves, Appl. Math. Lett., 12,115-119, (1999).

[7] D.J. Unger, Developable surfaces in elastoplastic fracture mechanics, Int. J. Fract. 50, 33-38, (1991).

[8] D. Latifi, A. Razavi, Inextensible flows of curves in Minkowskian Space, Adv. Studies Theor. Phys. 2(16), 761-768, (2008).

[9] E. Molnar, The projective interpretation of the eight 3-dimensional homogeneous geometries, Beitrage zur Algebra und Geometrie Contributions to Algebra and Geometry, 38 (2), 261-288, (1997).

[10] G. Chirikjian, J. Burdick, A modal approach to hyper-redundant manipulator kinematics, IEEE Trans. Robot. Autom. 10,343-354, (1994).

[11] H. Öztekin and H. Gün Bozok, Inextensible flows of curves according to Sabban frame in pseudo-Galilean space $G_{3}^{1}$, i-managers Journal on Mathematics, Vol.2, N.1, pp.1-12, (2013).

[12] H. Mochiyama, E. Shimemura, H. Kobayashi, Shape control of manipulators with hyper degrees of freedom, Int. J. Robot.Res.,18, 584-600, (1999).

[13] H.Q. Lu, J.S. Todhunter, T.W. Sze, Congruence conditions for nonplanar developable surfaces and their application to surface recognition, CVGIP,Image Underst. 56, 265-285, (1993).

[14] I.M. Yaglom, A Simple Non-Euclidean Geometry and Its Physical Basis, Springer-Verlag, New York, (1979).

[15] I. Kamenarovic, Existence Theorems for Ruled Surfaces in the Galilean Space $G_{3}$. Rad HAZU Math. 10, 183-196,(1991).

[16] J. Koenderink, Solid shape, MIT Press, Cambridge, MA, (1990).

[17] M. Ergüt, E. Turhan, T. Körpinar, Characterization of inextensible flows of spacelike curves with Sabban frame in $S_{1}^{2}$, Bol. Soc. Paran. Mat. 31(2), 47-53, (2013).

[18] M. Kass, A. Witkin, D. Terzopoulos, Snakes: active contour models, in: Proc. 1st Int. Conference on Computer Vision, 259-268 (1987).

[19] M. Desbrun, M.-P. Cani-Gascuel, Active implicit surface for animation, in: Proc. Graphics Interface-Canadian Inf. Process. Soc., 143-150, (1998).

[20] O. Röschel, Die Geometrie des Galileischen Raumes, Habilitationsschrift, Leoben, (1984).

[21] Z. M. Sipus, Ruled Weingarten surfaces in the Galilean space, Periodica Mathematica Hungarica, 56(2), 213-225, (2008).

Osmaniye Korkut Ata university, Department of Mathematics, 80000, Osmaniye, TURKey

E-mail address: hulyagun@osmaniye.edu.tr 